\title{
THE LEVY CONCENTRATION PHENOMENON FOR SPECIAL FUNCTIONS ON RANK-ONE SYMMETRIC SPACES*
}

\author{
MAUNG MIN-OO ${ }^{\dagger}$ AND JOHN A. TOTH ${ }^{\ddagger}$
}

1. Introduction. Paul Levy discovered the surprising phenomenon of measure concentration of Lipschitz maps for certain sequences of spaces as the dimension goes to infinity. This has been explored further in a geometrical framework by M.Gromov and V.Milman [GM][G][MS]. The main objective of this paper is to study in some detail this measure concentration phenomenon for compact, rank-one symmetric spaces. In particular, we will investigate the role of zonal eigenfunctions of the Laplacian and show that (see Theorems 1 and 2) the push-forward measures of a wide class of observables associated with these functions exhibit a much stronger concentration than the estimate one gets from the isoperimetric inequality of Gromov and Levy [G][MS].

Let $\left(M^{n}, g\right)$ be a compact, $n$-dimensional, $C^{\infty}$ Riemannian manifold and $d \mu_{n}$ be the associated $n$-dimensional measure. We will denote the normalized probability measure $\left(\operatorname{vol}\left(M^{n}, g\right)\right)^{-1} d \mu_{n}$ by $\hat{d} \mu_{n}$. Given a map $f: M^{n} \rightarrow \mathbb{R}$, we define:

$$
\|f\|_{L i p}:=\|f\|_{\infty}+\sup _{x \neq y} \frac{|f(x)-f(y)|}{d(x, y)} .
$$

We say that a sequence of functions $f_{n}: M^{n} \rightarrow \mathbb{R}$ is in $\operatorname{Lip}^{1}\left(M^{n}\right)$ (ie. $f_{n}$ is 1Lipschitz) if $f_{n} \in \operatorname{Lip}\left(M^{n}\right)$ and there exists $\kappa>0$ independent of the dimension, $n$, such that $\left\|f_{n}\right\|_{L i p} \leq \kappa$ for all $n$. In order to describe our results in greater detail, we start with a very simple observation:

Lemma 1. Let $\left(M^{n}, g\right)$ be a compact, $C^{\infty}$ Riemannian manifold with normalized measure $\hat{d} \mu_{n}$ and let $f_{n}: M^{n} \rightarrow \mathbb{C}$ a Lipschitz map with $f_{n} \in \operatorname{Lip}^{1}\left(M^{n}\right)$ and $\int_{M} f_{n} d \mu_{n}=0$. Then, for any test function, $g \in C_{0}^{\infty}(\mathbb{R})$, we have:

$$
I_{f_{n}}(g):=\int_{\mathbb{R}} g\left(f_{n}\right)_{*} \hat{d} \mu_{n}=g(0)+E(n)
$$

with error estimate:

$$
|E(n)| \leq \frac{C(g)}{\lambda_{1}}
$$

where $\lambda_{1}$ is the first non-zero eigenvalue of the Laplacian.

Proof. Make a second-order Taylor expansion:

$$
g\left(f_{n}(x)\right)=g(0)+g^{\prime}(0) f_{n}(x)+\mathcal{O}(1) f_{n}^{2}(x) .
$$

Since $\int_{M} f_{n} d \mu=0$, it follows that

$$
\int_{M} g\left(f_{n}(x)\right) \hat{d} \mu_{n}(x)=g(0)+\mathcal{O}(1) \int_{M} f_{n}^{2} \hat{d} \mu_{n}
$$

\footnotetext{
*Received August 25, 1999.

† Department of Mathematics and Statistics, McMaster University, Hamilton, Ontario, Canada, L8S 4K1 (minoo@mcmail.cis.mcmaster.ca). Supported in part by NSERC Grant OGP7873-99.

$\ddagger$ Department of Mathematics and Statistics, McGill University, 805 Sherbrooke St. West, Montreal, Canada, H3A 2K6 (toth@math.mcgill.ca). Supported in part by NSERC Grant OGP0170280.
} 
The lemma then follows from the Poincaré inequality:

$$
\int_{M}\left|f_{n}\right|^{2} \hat{d} \mu_{n} \leq \frac{1}{\lambda_{1}} \int_{M}\left\|\nabla f_{n}\right\|^{2} \hat{d} \mu_{n} \leq \frac{1}{\lambda_{1}} .
$$

Suppose we now also assume that

$$
\operatorname{Ric}\left(M^{n}, g\right) \geq(n-1) k>0 .
$$

Then, by the theorem of Lichnerowicz, $\lambda_{1} \geq k n$, and combined with Lemma 1 this in turn implies that

$$
\int_{\mathbb{R}} g\left(f_{n}\right)_{*} \hat{d} \mu_{n}=g(0)+\mathcal{O}\left(n^{-1}\right)
$$

for an arbitrary 1-Lipschitz map, $f_{n}$. By choosing $f_{n}$ to be the first 1-Lipschitz Laplace eigenfunction on $\mathbb{S}^{n}$ (see section 2), it is easily seen that the estimate in (2) cannot be improved for general observables. However, when $f_{n}$ is a 1-Lipschitz eigenfunction of the Laplacian, $-\Delta$, with eigenvalue, $\lambda_{k}$, Green's Theorem implies that, for any $g \in C_{0}^{\infty}(\mathbb{R})$,

$$
\int_{\mathbb{R}} g\left(f_{n}\right)_{*} \hat{d} \mu_{n}=g(0)+\mathcal{O}\left(\lambda_{k}^{-1}\right)
$$

Thus, in particular, for large enough $\lambda_{k}$ the estimate in (3) is better than (2).

Such dimensional concentration results are well-known (see [MS,GM,GLP]). Although Levy-Milman-Gromov results are mainly concerned with large deviation estimates, and we compute the second moment in Lemma 1, we will nevertheless refer to (2) as the LMG-estimate.

The plan of the paper is as follows: In sections 2 and 3, we show that (see Theorem 1) for Lipschitz-normalized, zonal eigenfunctions of the Laplacian on rank-one symmetric spaces, the LMG estimate can be improved significantly. This is consistent with (3), but the precise estimates involve a detailed study of the second moments appearing in the error terms and in fact, turn out to be much better than the naive prediction in (3).

In section 4, we show that (see Theorem 2) the error term in the LMG estimate can be improved for a large class of 1-Lipschitz functions including many Laplace eigenfunctions that are not radial (Corollary 1).

Although we will not pursue this here, we should point out that this dimensional concentration phenomena for zonals is really a problem in semiclassical analysis. In particular, the eigenfunction equations (5), (11) and (19) are all $\hbar$-differential equations where $\hbar^{-1}=n$, the dimensional parameter of the base rank-one space. Thus, the content of Theorem 1 is an estimate for the second moments of Lipschitz-normalized, semi-excited states associated with these equations. The question we are dealing with here is dual to the problem one usually studies in semiclassical analysis; namely, we consider sequences of 1-Lipschitz zonal eigenfunctions, where the base space (ie. the rank-one manifold) varies in dimension. This connection with semiclassical analysis seems to extend to more general situations.

One can also naturally consider dimensional asymptotics for compact, semi-simple Lie groups and higher-rank symmetric spaces. It would be of interest to establish sharp estimates for the dimensional measure concentration of special observables such as spherical functions (the analogues of the zonals) on such spaces. We hope to pursue all these questions in future work. 
2. Explicit calculations for the first eigenfunction. Before we investigate the dimensional asymptotics of general zonal eigenfunctions of the Laplacian for rank one symmetric spaces, $M^{n}=G / K$, we will begin with the first non-trivial zonal eigenfunction. The higher zonal functions are polynomials in the first zonal eigenfunction and we will treat them in the next section. In this section, we will compute all moments of the push-forward measure by the first non-trivial eigenfunction for compact, rank-one symmetric spaces.

\section{Case 0.}

$S^{n}$ with the round metric of sectional curvature $K=1$. The volume form in geodesic polar coordinates is given by $[\mathrm{BGM}][\mathrm{H}]$ :

$$
d \mu_{n}=(\sin r)^{n-1} d r d \theta
$$

where $d \theta$ is the volume form of the unit sphere $S^{n-1}$ and $0 \leq r \leq \pi$. Since the logarithmic derivative of $(\sin r)^{n-1}$ is $(n-1) \cot r$, we have the following expression for the Laplacian acting on a radial function $\phi(r)$ :

$$
\Delta \phi(r)=\phi^{\prime \prime}(r)+(n-1) \cot r \phi^{\prime}(r) .
$$

It follows that $\phi_{1}(r)=-\cos r$ satisfies $-\Delta \phi_{1}(r)=n \phi_{1}(r)$ and is a zonal eigenfunction for the lowest eigenvalue $n$ which is 1-Lipschitz. The volume form can be expressed in terms of $\phi_{1}$ as:

$$
d \mu_{n}=\left(1-\phi_{1}^{2}\right)^{\frac{n}{2}-1} d \phi_{1} d \theta
$$

where $\phi_{1}$ ranges in the interval $[-1,+1]$. Therefore, if we normalize the push-forward measure $\left(\phi_{1}\right)_{*} d \mu_{n}$, we get the following measure supported in the interval $[-1,+1] \subset$ $\mathbb{R}$ :

$$
\hat{d} \mu_{1}=\frac{1}{I_{n}}\left(1-\phi_{1}^{2}\right)^{\frac{n}{2}-1} d \phi_{1}
$$

where $I_{n}=\int_{-1}^{+1}\left(1-x^{2}\right)^{\frac{n}{2}-1} d x=B\left(\frac{n}{2}, \frac{1}{2}\right)$. This is a probability measure on $\mathbb{R}$ with mean zero and variance:

$$
\frac{1}{I_{n}} \int_{-1}^{+1} x^{2}\left(1-x^{2}\right)^{\frac{n}{2}-1} d x=\frac{1}{n+1}
$$

which is of order $\mathcal{O}\left(n^{-1}\right)$.

The estimate in (8) holds for any eigenfunction in the first eigenspace, since, for the sphere, they are all zonal with respect to a suitable pole.

If we compute the higher moments, we find that the odd moments vanish and the $2 p$-th moment is:

$$
\frac{1}{I_{n}} \int_{-1}^{+1} x^{2 p}\left(1-x^{2}\right)^{\frac{n}{2}-1} d x=\frac{1 \cdot 3 \cdots(2 p-1)}{(n+1)(n+3) \cdots(n+2 p-1)}
$$

which is of order $\mathcal{O}\left(n^{-p}\right)$.

\section{Case 1.}

$\mathbb{C} P^{n}$ with the Fubini-Study metric with sectional curvature satisfying $1 \leq K \leq 4$. The volume form of this metric in geodesic polar coordinates is given by $[\mathrm{BGM}][\mathrm{H}]$ :

$$
d \mu_{n}=\frac{1}{2}(\sin r)^{2 n-2} \sin 2 r d r d \theta=(\sin r)^{2 n-1} \cos r d r d \theta
$$


where $d \theta$ is the volume form of the unit standard sphere and $0 \leq r \leq \frac{\pi}{2}$. Since the logarithmic derivative of $(\sin r)^{2 n-2} \sin 2 r$ is $(2 n-2) \cot r+2 \cot 2 r$, we have the following expression for the Laplacian acting on a radial function $\phi(r)$ :

$$
-\Delta \phi(r)=\phi^{\prime \prime}(r)+((2 n-2) \cot r+2 \cot 2 r) \phi^{\prime}(r) .
$$

It follows now by a direct calculation that

$$
\phi_{1}(r)=\frac{1}{n+1}-(\cos r)^{2}=(\sin r)^{2}-\frac{n}{n+1}=-\left(\frac{1}{2} \cos 2 r+\frac{n-1}{n+1}\right)
$$

satisfies

$$
-\Delta \phi_{1}(r)=4(n+1) \phi_{1}(r)
$$

and hence is a zonal eigenfunction for the lowest eigenvalue. Note that, we have normalized the eigenfunction $\phi_{1}$ to be 1-Lipschitz. as:

Since $\sin ^{2} r=\phi_{1}+\frac{n}{n+1}$ and $d \phi_{1}=2 \sin r \cos r$, the volume form can be expressed

$$
d \mu_{n}=\frac{1}{2}\left(\phi_{1}+\frac{n}{n+1}\right)^{n-1} d \phi_{1} d \theta
$$

where $\phi_{1}$ ranges in the interval $\left[-\frac{n}{n+1}, \frac{1}{n+1}\right]$. Therefore, if we normalize the pushforward measure $\left(\phi_{1}\right)_{*} d \mu_{n}$ to have unit total mass, we get the following measure supported in the interval $\left[-\frac{n}{n+1}, \frac{1}{n+1}\right] \subset \mathbb{R}$ :

$$
\hat{d} \mu_{1}=n\left(\phi_{1}+\frac{n}{n+1}\right)^{n-1} d \phi_{1} \text {. }
$$

This is a probability measure on $\mathbb{R}$ with mean zero, since $\int \phi_{1} d \mu_{n}=0$. The variance is:

$$
n \int_{-\frac{n}{n+1}}^{\frac{1}{n+1}} \phi_{1}^{2}\left(\phi_{1}+\frac{n}{n+1}\right)^{n-1} d \phi_{1}=\frac{n}{(n+1)^{2}(n+2)}
$$

So, the variance is of order $\mathcal{O}\left(n^{-2}\right)$ and is much sharper than the case of the sphere where it is $\mathcal{O}\left(n^{-1}\right)$, even for the first eigenfunction. If we compute the higher moments we find that the $p^{\text {th }}$ moment is:

$$
n \int_{-\frac{n}{n+1}}^{\frac{1}{n+1}} \phi_{1}^{p}\left(\phi_{1}+\frac{n}{n+1}\right)^{n-1} d \phi_{1}=n \int_{0}^{1}\left(x-\frac{n}{n+1}\right)^{p} x^{n-1} d x
$$

which is a rational expression in $n$ of order $\mathcal{O}\left(n^{-p}\right)$.

At this point, we would like to remark that the large deviation estimate for this pushforward measure trivially satisfies the Levy-type inequality:

$$
\int_{0}^{1-\epsilon} d\left(x^{n}\right)=(1-\epsilon)^{n} \leq e^{-n \epsilon}
$$

However, $\epsilon$ is measured in terms of the values of the first eigenfunction $\phi_{1}$ and not with respect to the distance function $r$. 


\section{Case 2.}

$\mathbb{H} P^{n}$ with the symmetric metric of sectional curvature $1 \leq K \leq 4$. The volume form of this metric in geodesic polar coordinates is given by $[\bar{B} G M][\mathrm{H}]$ :

$$
d \mu_{n}=\frac{1}{8}(\sin r)^{4 n-4}(\sin 2 r)^{3} d r d \theta=(\sin r)^{4 n-1}(\cos r)^{3} d r d \theta
$$

where $d \theta$ is the volume form of the unit sphere $S^{4 n-1}$ and $0 \leq r \leq \frac{\pi}{2}$. The Laplacian acting on a radial function $\phi(r)$ is given by:

$$
-\Delta \phi(r)=\phi^{\prime \prime}(r)+((4 n-4) \cot r+6 \cot 2 r) \phi^{\prime}(r) .
$$

Again, by a direct calculation we find that

$$
\phi_{1}(r)=(\cos r)^{2}-\frac{1}{n+1}=\frac{1}{2} \cos 2 r+\frac{n-1}{n+1}
$$

satisfies

$$
-\Delta \phi_{1}(r)=8(n+1) \phi_{1}(r)
$$

and hence, is a 1-Lipschitz zonal eigenfunction. Since $\sin ^{2} r=\phi_{1}+\frac{n}{n+1}$ and $d \phi_{1}=$ $2 \sin r \cos r$, the volume form can be expressed as:

$$
d \mu_{n}=\frac{1}{2}\left[\left(\phi_{1}+\frac{n}{n+1}\right)^{2 n-1}-\left(\phi_{1}+\frac{n}{n+1}\right)^{2 n}\right] d \phi_{1} d \theta
$$

where $\phi_{1}$ ranges in the interval $\left[-\frac{n}{n+1}, \frac{1}{n+1}\right]$. Therefore, the normalized push-forward probability measure is given by:

$$
\hat{d} \mu_{1}=2 n(2 n+1)\left[\left(\phi_{1}+\frac{n}{n+1}\right)^{2 n-1}-\left(\phi_{1}+\frac{n}{n+1}\right)^{2 n}\right] d \phi_{1}
$$

where $\phi_{1}$ is supported in the interval $\left[-\frac{n}{n+1}, \frac{1}{n+1}\right] \subset \mathbb{R}$. Here, $\hat{d} \mu_{1}$ is a probability measure on $\mathbb{R}$ with mean zero and variance:

$$
\int_{\mathbb{R}} \phi_{1}^{2} \hat{d} \mu_{1}=2 n(2 n+1) \int_{0}^{1} x^{2}\left(x^{2 n-1}-x^{2 n}\right) d x-\frac{n^{2}}{(n+1)^{2}}=\frac{n}{(n+1)^{2}(2 n+3)} .
$$

The variance is again of order $\mathcal{O}\left(n^{-2}\right)$, sharper than the case of the sphere. As in the case of $\mathbb{C} P^{n}$, we find that the $p^{\text {th }}$ moment:

$$
\int_{\mathbb{R}} \phi_{1}^{p} \hat{d} \mu_{1}=2 n(2 n+1) \int_{0}^{1}\left(x-\frac{n}{n+1}\right)^{p}\left(x^{2 n-1}-x^{2 n}\right) d x
$$

is a rational expression in $n$ of order $\mathcal{O}\left(n^{-p}\right)$.

3. Dimensional asymptotics for higher zonal eigenfunctions. Let $M$ be a rank-one symmetric space $G / K$ where $K$ is the isotropy group at the point $p_{0} \in M$. Let $K_{O}$ be the identity component of $K$ and $V$ be a finite-dimensional subspace of $C^{\infty}(M)$ invariant under $l(g)$, where

$$
l(g) f(x):=f\left(g^{-1} x\right)
$$


for all $g \in G$ and $x \in M$. The space of zonal functions [H] centered at $p_{0}$ is then defined to be

$$
\mathcal{Z}(V)=\left\{v \in V ; l(k) v=v \text { for all } k \in K_{0}\right\} .
$$

In particular, one can take $V$ to be the eigenspace

$$
V_{\lambda}=\left\{u \in C^{\infty}(M) ; \Delta u=-\lambda u\right\} .
$$

It is well-known that the action

$$
l(g): V_{\lambda} \rightarrow V_{\lambda}
$$

is irreducible and moreover, the assumption that $M$ is a rank-one symmetric space ensures that

$$
\operatorname{dim} \mathcal{Z}\left(V_{\lambda}\right)=1
$$

Furthermore, it follows readily from the definition of $\mathcal{Z}\left(V_{\lambda}\right)$ that all functions $\phi \in$ $\mathcal{Z}\left(V_{\lambda}\right)$ are radial; that is $\phi(x)=\phi(|x|)$ where, $|x|=d\left(x, p_{0}\right)$.

Our objective is to study in some detail the LMG estimate (2) for rank-one symmetric spaces, $M^{n}=G / K$, where the observable is a zonal eigenfunction of the Laplacian. To wit, let $\phi_{m}(r)$ be the 1-Lipschitz zonal eigenfunction of the Laplacian, $-\Delta$, with eigenvalue, $\lambda_{m}$. We will now show that these functions have much sharper concentration properties in the dimensional limit than the general LMG estimate predicts.

We begin with the case of $\mathbb{S}^{n}$ normalized so that $K=1$. Recall, the Poisson kernel for the unit ball $B=\left\{x \in \mathbb{R}^{n+1} ;|x|^{2} \leq 1\right\}$ is:

$$
P(x, y)=\frac{1}{\operatorname{vol}\left(\mathbb{S}^{n}\right)} \frac{1-|x|^{2}}{|x-y|^{n+1}} .
$$

Let $\mathcal{Z}_{m}(x, y)$ be the $m$-th zonal harmonic on $\mathbb{S}^{n}$ centered at $y \in \mathbb{S}^{n}$. The reproducing property $[F]$ of these functions implies that:

$$
P(x, y)=\sum_{m=0}^{\infty}|x|^{m} \mathcal{Z}_{m}\left(\frac{x}{|x|}, y\right) .
$$

By expanding $P(x, y)$, we get the following explicit formulas for the zonal harmonic of degree $m$ centered at $y_{0}=(0,,, 0,1) \in \mathbb{R}^{n+1}$ :

$$
\mathcal{Z}_{m}\left(x, y_{0}\right)=\sum_{k=0}^{\left[\frac{m}{2}\right]} c_{k}(n) x_{n+1}^{m-2 k}|x|^{2 k}
$$

Here, $\left(x_{1}, \ldots, x_{n+1}\right) \in \mathbb{R}^{n+1}$ and

$$
c_{k}(n)=(-1)^{k} \frac{(n+2 m-1)(n+1)(n+3) \cdots(n+2 m-2 k-3)}{2^{k} k !(2 m-k) !} .
$$

We henceforth assume that $m$ is fixed independent of the dimension, $n$. Then,

$$
c_{k}(n) \sim n^{m-k}
$$


Let $(r, \theta) \in \mathbb{R}^{+} \times \mathbb{S}^{n-1}$ denote geodesic polar coordinates centered at $(0, \ldots, 0,1) \in$ $\mathbb{R}^{n+1}$. Since $x_{n+1}=\cos r=\phi_{1}(r)$, it then follows that the Lipschitz-1 eigenfunction, $\phi_{m}(r)$, corresponding to $\mathcal{Z}_{m}\left(x, y_{0}\right)$ is of the form:

$(27) \phi_{m}(r)=C_{m}(n)\left[\left(\phi_{1}(r)\right)^{m}+\frac{c_{1}(n)}{c_{0}(n)}\left(\phi_{1}(r)\right)^{m-2}+\ldots+\frac{c_{m}(n)}{c_{0}(n)}\left(\phi_{1}(r)\right)^{m-2\left[\frac{m}{2}\right]}\right]$,

where, $C_{m}(n)=C_{m}+\mathcal{O}\left(n^{-1}\right)$ and

$$
\frac{c_{k}(n)}{c_{0}(n)}=\mathcal{O}\left(n^{-k}\right)
$$

Since $\int_{\mathbb{S}^{n}} \phi_{m} d \mu_{n}=0$, we must compute the second moment of $\phi_{m}$. To do this, note that by our moment computations for $\phi_{1}$ in section 2 , we have for $0 \leq k, l \leq\left[\frac{m}{2}\right]$,

$$
\left(\frac{c_{k}(n)}{c_{0}(n)}\right)\left(\frac{c_{l}(n)}{c_{0}(n)}\right) \int_{\mathbb{R}}\left(\phi_{1}\right)^{m-2 k}\left(\phi_{1}\right)^{m-2 l} \hat{d} \mu_{1}=\mathcal{O}\left(n^{-m}\right)
$$

It follows that:

$$
\int_{\mathbb{R}} \phi_{m}^{2} \hat{d} \mu_{1}=\mathcal{O}\left(n^{-m}\right)
$$

Now consider complex projective space, $\mathbb{C} P^{n}$, normalized so that $1 \leq K \leq 4$. Let $\mathbb{B}=\left\{z \in \mathbb{C}^{n+1} ;|z|^{2} \leq 1\right\}$ and denote the space of harmonic polynomials in $\mathbb{B}$ of degree $p$ in $z$ and degree $q$ in $\bar{z}$ by $\mathcal{H}^{p, q}$. The zonal eigenfunction in this vector space will be denoted by $H^{p, q}$. Since we are interested in $\mathbb{C} P^{n}$, we need only consider those zonals which are invariant under the circle action:

$$
e^{i \theta} \cdot\left(z_{1}, \ldots, z_{n+1}\right)=\left(e^{i \theta} z_{1}, \ldots, e^{i \theta} z_{n+1}\right)
$$

Consequently, for $m$ fixed independent of the dimension, $n$, we put $p=q=m$. Then, for fixed $\zeta \in \partial \mathbb{B}$, consider $H^{m, m}(z \cdot \bar{\zeta})$. It is readily verified that [Ko]:

$$
H^{m, m}(z \cdot \bar{\zeta})=C(m, n) \cdot P_{m}^{\left(\frac{n}{2}-1,0\right)}\left(2|z \cdot \bar{\zeta}|^{2}-1\right) .
$$

Here, $C(m, n)$ is a constant and $P_{m}^{(\alpha, \beta)}$ denotes the Jacobi polynomial [AS] of degree $m$ with indices $(\alpha, \beta)$ :

$$
P_{m}^{(\alpha, \beta)}(t)=\frac{1}{2^{m}} \sum_{k=0}^{m}\left(\begin{array}{c}
m+\alpha \\
k
\end{array}\right)\left(\begin{array}{c}
m+\beta \\
m-k
\end{array}\right)(t-1)^{m-k}(1+t)^{k}
$$

Fix $\zeta=(0,0, \ldots, 0,1) \in \mathbb{C}^{n+1}$ and consider geodesic polar coordinates $(r, \theta) \in$ $\mathbb{R}_{+} \times \mathbb{S}^{n-1}$ centered at $\zeta$. In terms of these coordinates,

$$
\left|z_{n+1}\right|^{2}=(\cos r)^{2} \text {. }
$$

So, the m-th zonal eigenfunction in (31) is, up to multiplicative constant, the function

$$
P_{m}^{\left(\frac{n}{2}-1,0\right)}\left(2 \cos ^{2} r-1\right) \text {. }
$$


Next, we must Lipschitz-normalize this function. To do this, note that the expansion in (32) gives:

$$
P_{m}^{\left(\frac{n}{2}-1,0\right)}\left(2 \cos ^{2} r-1\right)=\sum_{k=0}^{m} c_{k}(n)\left(\sin ^{2} r\right)^{m-k}\left(\cos ^{2} r\right)^{k},
$$

where, $c_{k}(n) \sim n^{k}$ as $n \rightarrow \infty$. So, to Lipschitz normalize this function, we divide by $c_{0}(n) \sim n^{m}$. Consequently, the $m$-th, 1-Lipschitz zonal eigenfunction is of the form:

$$
\phi_{m}(r)=\sum_{k=0}^{m} \frac{c_{k}(n)}{c_{0}(n)}\left(\sin ^{2} r\right)^{m-k}\left(\cos ^{2} r\right)^{k} .
$$

Again, we wish to compute the second moment $\int_{\mathbb{R}} \phi_{m}^{2} \hat{d} \mu_{1}$. To do this, note that, for $0 \leq k, l \leq m$ :

$$
\begin{gathered}
\left(\frac{c_{k}(n)}{c_{0}(n)}\right)\left(\frac{c_{l}(n)}{c_{0}(n)}\right) \int_{\mathbb{R}}(\sin r)^{2(2 m-k-l)}(\cos r)^{2(k+l)} \hat{d} \nu \\
\leq\left(\frac{c_{k}(n)}{c_{0}(n)}\right)\left(\frac{c_{l}(n)}{c_{0}(n)}\right) \int_{\mathbb{R}}\left(\phi_{1}-\frac{1}{n+1}\right)^{2(k+l)} \hat{d} \mu_{1}=\mathcal{O}\left(n^{-2 m}\right) .
\end{gathered}
$$

The last line (37) follows readily from the estimate $|\sin r| \leq 1$, the binomial expansion and our estimates for the moments of $\phi_{1}$ in section 2. So, we have shown that:

$$
\int_{\mathbb{R}} \phi_{m}^{2} \hat{d} \mu_{1}=\mathcal{O}\left(n^{-2 m}\right) .
$$

Finally, we turn to the case of the quaternionic projective spaces, $\mathbb{H} P^{n}$. Here, one can make a direct change of variables in the eigenfunction equation [Ko] to transform it to a canonical equation for orhogonal polynomials. The end result is that

$$
\phi_{m}(r)=C(m, n) P_{m}^{\left(\frac{n}{2}-1,1\right)}\left(2 \cos ^{2} r-1\right) .
$$

As the estimates are very similar to the case of $\mathbb{C} P^{n}$, we leave the details to the reader.

Taking into account the Taylor expansion in Lemma 1, we have now proved:

THEOREM 1. Let $\phi_{m}(r)$ be the $m$-th, 1-Lipschitz, zonal eigenfunction of the Laplacian on the rank-one symmetric space $M^{n}=G / K$ and let $g \in C_{0}^{\infty}(\mathbb{R})$. Suppose $m$ is arbitrarily large but fixed independent of the dimension, $n$. Then, for $M^{n}=$ $\mathbb{C} P^{n}, \mathbb{H} P^{n}$ :

$$
\int_{\mathbb{R}} g(t)\left(\phi_{m}\right)_{*} \hat{d} \mu_{n}(t)=g(0)+\mathcal{O}\left(n^{-2 m}\right)
$$

whereas, for $M^{n}=\mathbb{S}^{n}$ :

$$
\int_{\mathbb{R}} g(t)\left(\phi_{m}\right)_{*} \hat{d} \mu_{n}(t)=g(0)+\mathcal{O}\left(n^{-m}\right)
$$

REMARK. It follows readily from the above analysis that the estimates in Theorem 2 are valid for sequences $\phi_{m(n)} ; n=1,2, \ldots$ with $m(n) \sim n^{\alpha}$, provided $0 \leq \alpha<1$. Thus, in particular, when $m(n)=n^{\alpha}$, the error terms in Theorem 1 are $\mathcal{O}\left(n^{-n^{\alpha}+2 \alpha}\right)$ and $\mathcal{O}\left(n^{-2 n^{\alpha}+2 \alpha}\right)$ respectively. 
4. The general estimate. In the last section, we showed that for the complex and quaternionic projective spaces, the 1-Lipschitz zonal eigenfunctions of the Laplacian have stronger dimensional concentration than the LMG estimate predicts. Our objective here is to extend this refined concentration estimate to a wider class of 1-Lipschitz observables containing the zonal eigenfunctions.

Consider the complex vector space, $V_{\lambda_{1}}$, of eigenfunctions of the Laplacian, $\Delta$, with eigenvalue, $\lambda_{1}$. There is a natural space of linearly independent, zonal eigenfunctions in $V_{\lambda_{1}}$, which we will denote by $\mathcal{Z}\left(M^{n} ; \lambda_{1}\right)$. For instance, on the sphere, $\mathbb{S}^{n}$

$$
\mathcal{Z}\left(\mathbb{S}^{n} ; \lambda_{1}\right):=\left\{x_{1}, \ldots, x_{n+1}\right\}
$$

where for $k=1, \ldots, n+1, x_{k}$ denote the restrictions to $\mathbb{S}^{n}$ of the standard coordinate functions on $\mathbb{R}^{n+1}$. On complex projective space,

$$
\mathcal{Z}\left(\mathbb{C} P^{n} ; \lambda_{1}\right):=\left\{\left|z_{k}\right|^{2}-\frac{1}{n+1}\right\}_{1 \leq k \leq n+1}
$$

where $\left[z_{k}\right]$ denote the standard homogeneous coordinates on $\mathbb{C}^{n+1}$ restricted to $\mathbb{S}^{2 n+1}$. The space $\mathcal{Z}\left(\mathbb{H} P^{n} ; \lambda_{1}\right)$ is identical to (40), provided we replace complex multiplication by quaternionic multiplication. Recall, we say that $f_{n} \in \operatorname{Lip}^{1}\left(M^{n}\right)$, provided $f_{n} \in$ $\operatorname{Lip}\left(M^{n}\right)$ and there exists a constant, $\kappa>0$, independent of the dimension, $n$, such that $\left\|f_{n}\right\|_{L i p} \leq \kappa$.

Definition. $\mathcal{I}^{*}\left(M^{n}\right)$ is defined to be the finitely-generated ideal over $\operatorname{Lip}^{1}\left(M^{n}\right)$, generated by the elements of $\mathcal{Z}\left(M^{n} ; \lambda_{1}\right)$.

In the following, we will, without loss of generality, always assume that $\int f_{n}=0$. Moreover, given geodesic spherical coordinates $(r, \theta)$ centered at a point $p \in M$, we define the spherical mean $\mathcal{M}_{f_{n}}$ by:

$$
\mathcal{M}_{f_{n}}(r):=\int_{\mathbb{S}^{2 n-1}} f_{n}(r, \omega) \hat{d} \omega
$$

We can now state our result:

Theorem 2. (i) Let $M^{n}=G / K$ be a rank-one symmetric space and $g \in C_{0}^{\infty}(\mathbb{R})$. Suppose $f_{n} \in \mathcal{I}\left(M^{n}\right)$ is generated by a zonal eigenfunction, $\phi(z, \bar{z})=\left|z_{k}\right|^{2}-(n+1)^{-1}$; that is $f_{n}(z, \bar{z})=\phi_{1}(z, \bar{z}) \cdot h(z, \bar{z} ; n)$. Assume moreover, that there exist a constant $C>0$ independent of $n$ such that

$$
\left\|\mathcal{M}_{|h|^{2}}\right\|_{\infty} \leq C
$$

When $M^{n}=\mathbb{C} P^{n}, \mathbb{H} P^{n}$ :

$$
\int_{\mathbb{R}} g(t)\left(f_{n}\right)_{*} \hat{d} \mu_{n}(t)=g(0)+\mathcal{O}\left(n^{-2}\right) .
$$

as $n \rightarrow \infty$. On the other hand, when $M^{n}=\mathbb{S}^{n}$ :

$$
\int_{\mathbb{R}} g(t)\left(f_{n}\right)_{*} \hat{d} \mu_{n}(t)=g(0)+\mathcal{O}\left(n^{-1}\right)
$$


Moreover, these estimates are sharp.

(ii) Let $f_{n} \in \mathcal{I}\left(\mathbb{C} P^{n}\right)$ with $f_{n}=\sum_{j=1}^{n+1}\left|z_{j}\right|^{2} \cdot h_{j}(z, \bar{z})$. Suppose that, for $j=1, \ldots, n+1$, there exists a constant $C>0$ independent of the dimension, such that $h_{j} \in \operatorname{Lip}^{1}\left(M^{n}\right)$ satisfies:

$$
\sum_{j=1}^{n+1}\left\|h_{j}\right\|_{\infty}^{2} \leq C \text { and } \sum_{j=1}^{n+1}\left\|\nabla h_{j}\right\|_{\infty}^{2} \leq C n .
$$

Then, the estimates in part (i) are valid for these $f_{n}$ 's.

Proof of (i). To begin, we will assume that $M^{n}=\mathbb{C} P^{n}$ and without loss of generality, it suffices to assume that:

$$
f_{n}(z, \bar{z})=\left|z_{n+1}\right|^{2} \cdot h(z, \bar{z} ; n) .
$$

Given $g \in C_{0}^{\infty}(\mathbb{R})$, we must give an asymptotic expansion for the push-forward integral:

$$
I_{g}\left(f_{n}\right):=\int_{\mathbb{R}} g(t)\left(f_{n}\right)_{*} \hat{d} \mu_{n}(t)=\int_{0}^{\pi / 2} \int_{\mathbb{S}^{2 n-1}} g\left(f_{n}(r, \theta)\right)(\cos r)(\sin r)^{2 n-1} \hat{d} \theta \hat{d} r .
$$

By a second-order Taylor expansion, we have for each $r \in[0, \pi / 2]$,

$$
\begin{aligned}
\int_{\mathbb{S}^{2 n-1}} g\left(f_{n}(r, \theta)\right) \hat{d} \theta= & g\left(\int_{\mathbb{S}^{2 n-1}} f_{n}(r, \omega) \hat{d} \omega\right) \\
& +\mathcal{O}_{g}(1) \int_{\mathbb{S}^{2 n-1}}\left|f_{n}(r, \theta)-\int_{\mathbb{S}^{2 n-1}} f_{n}(r, \omega) \hat{d} \omega\right|^{2} \hat{d} \theta .
\end{aligned}
$$

Denoting by $E(r ; n)$ the last term on the RHS of the above equation, we have by the Cauchy-Schwartz inequality,

$$
E(r ; n) \leq 2(\cos r)^{4} \int_{\mathbb{S}^{2 n-1}}|h(r, \theta ; n)|^{2} \hat{d} \theta \leq 2 C(\cos r)^{4}
$$

Note that by the estimate in (43),

$$
\frac{\int_{0}^{\pi / 2} E(r ; n)(\cos r)(\sin r)^{2 n-1} d r}{\int_{0}^{\pi / 2}(\cos r)(\sin r)^{2 n-1} d r} \leq 2 C\left(\frac{\int_{0}^{\pi / 2}(\cos r)^{5}(\sin r)^{2 n-3} d r}{\int_{0}^{\pi / 2}(\cos r)(\sin r)^{2 n-1} d r}\right)=\mathcal{O}\left(n^{-2}\right) .
$$

Recapping, we have so far shown that:

$$
I_{g}\left(f_{n}\right)=\frac{\int_{0}^{\pi / 2} g\left(\mathcal{M}_{f_{n}}(r)\right)(\cos r)(\sin r)^{2 n-1} d r}{\int_{0}^{\pi / 2}(\cos r)(\sin r)^{2 n-1} d r}+\mathcal{O}\left(n^{-2}\right) .
$$

Next, we integrate over the radial variable, $r$. To wit, consider the second-order Taylor expansion:

$$
g\left(\mathcal{M}_{f_{n}}(r)\right)=g(0)+g^{\prime}(0) \mathcal{M}_{f_{n}}(r)+\mathcal{O}_{g}(1) \mathcal{M}_{f_{n}}^{2}(r)
$$


Since by assumption, $\int f_{n}=0$, it follows that:

$$
I_{g}=g(0)+\mathcal{O}(1) \cdot\left(\frac{\int_{0}^{\pi / 2} \mathcal{M}_{f_{n}}^{2}(r)(\cos r)(\sin r)^{2 n-1} d r}{\int_{0}^{\pi / 2}(\cos r)(\sin r)^{2 n-1} d r}\right)+\mathcal{O}\left(n^{-2}\right)
$$

Again, by the Cauchy-Schwartz inequality,

$$
\left|\mathcal{M}_{f_{n}}(r)\right|^{2} \leq(\cos r)^{4}\left|\mathcal{M}_{|h|^{2}}(r)\right| \leq C(\cos r)^{4} .
$$

So, it follows that:

$$
\frac{\int_{0}^{\pi / 2} \mathcal{M}_{f_{n}}(r)^{2}(\cos r)(\sin r)^{2 n-1} d r}{\int_{0}^{\pi / 2}(\cos r)(\sin r)^{2 n-1} d r} \leq C\left(\frac{\int_{0}^{\pi / 2}(\cos r)^{5}(\sin r)^{2 n-1} d r}{\int_{0}^{\pi / 2}(\cos r)(\sin r)^{2 n-1} d r}\right)=\mathcal{O}\left(n^{-2}\right) .
$$

Therefore,

$$
I_{g}\left(f_{n}\right)=g(0)+\mathcal{O}\left(n^{-2}\right) .
$$

The analogous result for $\mathbb{H} P^{n}$ with $\mathcal{O}\left(n^{-2}\right)$ error term is proved in the same way. On the other hand, the corresponding result for $\mathbb{S}^{n}$ has an $\mathcal{O}\left(n^{-1}\right)$ error. Moreover, it follows by our earlier computations for the first, non-trivial, 1-Lipschitz eigenfunctions that all these estimates are sharp.

Proof of (ii). By the Taylor expansion in Lemma 1:

$$
\int_{\mathbb{R}} g(t)\left(f_{n}\right)_{*} \hat{d} \mu_{n}(t)=g(0)+E(n)
$$

where,

$$
|E(n)| \leq \frac{1}{4(n+1)} \int_{\mathbb{C P}^{n}}\left|\nabla f_{n}\right|^{2} \hat{d} \mu_{n}
$$

Clearly,

$$
\left|\nabla f_{n}\right|^{2} \leq 2 \sum_{j=1}^{n+1}\left[\left|z_{j}\right|^{4}\left|\nabla h_{j}\right|^{2}+2\left|h_{j}\right|^{2}\left(1-\left|z_{j}\right|^{2}\right)\left|z_{j}\right|^{2}\right]
$$

So, it follows that:

$$
|E(n)| \leq \frac{1}{2(n+1)} \cdot \sum_{j=1}^{n+1}\left(\left\|\nabla h_{j}\right\|_{\infty}^{2} \int_{\mathbb{C P}^{n}}\left|z_{j}\right|^{4} \hat{d} \mu_{n}+2\left\|h_{j}\right\|_{\infty}^{2} \int_{\mathbb{C} P^{n}}\left(1-\left|z_{j}\right|^{2}\right)\left|z_{j}\right|^{2} \hat{d} \mu_{n}\right) .
$$

Here,

$$
\int_{\mathbb{C P}^{n}}\left|z_{j}\right|^{4} \hat{d} \mu_{n}=\int_{\mathbb{R}}\left(\phi_{1}-\frac{1}{n+1}\right)^{2} \hat{d} \mu_{1}=\mathcal{O}\left(n^{-2}\right)
$$

by our computations in section 2 . On the other hand,

$$
\int_{\mathbb{C}^{n}}\left(1-\left|z_{j}\right|^{2}\right)\left|z_{j}\right|^{2} \hat{d} \mu_{n} \leq \int_{\mathbb{C}^{n}}\left|z_{j}\right|^{2} \hat{d} \mu_{n}=\int_{\mathbb{R}}\left(\phi_{1}-\frac{1}{n+1}\right) \hat{d} \mu_{1}=\mathcal{O}\left(n^{-1}\right) .
$$


Part (ii) of Theorem 2 follows.

REMARKs. (1) Note that part (i) of Theorem 2 shows that, $h(z, \bar{z} ; n)$ need not even be uniformly bounded as $n \rightarrow \infty$ for the $\mathcal{O}\left(n^{-2}\right)$ error to hold. All we need is that the second spherical mean $\mathcal{M}_{|h|^{2}}$ be uniformly bounded.

(2) Note that part (ii) of Theorem 1 extends in a straightforward fashion to both $\mathbb{H} P^{n}$ and $\mathbb{S}^{n}$. We simply replace $\left|z_{j}\right|^{2}$ by $x_{j}^{2}$ in the case of the sphere.

Suppose we now fix $k \in \mathbb{Z}^{+}$independent of the dimension, $n$, and consider the usual embedding $\iota: \mathbb{C} P^{k} \rightarrow \mathbb{C} P^{n}$ given by:

$$
\iota\left(\left[z_{1}, \ldots, z_{k+1}\right]\right)=\left[z_{1}, \ldots, z_{k+1}, 0, \ldots, 0\right] .
$$

Let $\psi_{m} \in V_{\lambda_{m}}\left(\mathbb{C} P^{k}\right)$ be a 1-Lipschitz Laplace eigenfunction on $\mathbb{C} P^{k}$ in the $m$-th eigenspace. We can naturally think of $\psi_{m}$ as a 1-Lipschitz Laplace eigenfunction on $\mathbb{C} P^{n}$ via the embedding, $\iota$. The following is an easy consequence of Theorem 2 (ii):

Corollary 1. Suppose $m \in \mathbb{Z}^{+}$and $k \in \mathbb{Z}^{+}$are fixed arbitrarily large, but independent of the dimensional parameter, $n$. Then, for arbitrary $g \in C_{0}^{\infty}(\mathbb{R})$ and $n$ sufficiently large,

$$
\int_{\mathbb{R}} g(t)\left(\psi_{m}\right)_{*} \hat{d} \mu_{n}(t)=g(0)+\mathcal{O}\left(n^{-2 m}\right)
$$

Proof. We will suppose that $\psi_{1} \in V_{\lambda_{1}}\left(\mathbb{C} P^{k}\right) \subset V_{\lambda_{1}}\left(\mathbb{C} P^{n}\right)$. Then, we can write:

$$
\psi_{1}(z, \bar{z})=\sum_{i, j=1}^{k+1} c_{i j} z_{i} \overline{z_{j}}
$$

where the $c_{i j}$ 's are complex numbers satisfying $\sum_{i=1}^{n+1} c_{i i}=0$ and $[z] \in \mathbb{S}^{2 n+1}$. The error term in the computation of the push-forward integral, $I_{\psi_{1}}(g)$, is just the second moment, $\int \psi_{1}^{2} \hat{d} \mu_{n}$. This consists of linear combinations of $(k+1)^{2}$ terms of the form:

$$
I_{i j k l}=\int_{\mathbb{C} P^{n}} z_{i} \overline{z_{j}} z_{k} \overline{z_{l}} \hat{d} \mu_{n} .
$$

Note that $I_{i j k l}=0$ unless, either $i=j$ and $k=l$, or $i=l$ and $j=k$. In both cases, we are reduced to assuming that $c_{i j}=0$ for $i \neq j$ and so, the estimate follows from Theorem 2 (ii), since the coefficients $c_{i i}$ are just constants which are independent of the dimension, $n$. The argument for the higher eigenfunctions, $\psi_{m}$, is very similar and is left to the reader. $\mathbb{S}^{n}$.

REMARK. There is a straightforward analogue of Corollary 1 for both $\mathbb{H} P^{n}$ and

\section{REFERENCES}

[AS] M. Abramovitz And R. Stegun, Handbook of Mathematical Functions, Dover, 1970.

[BGM] M. Berger, P. Gauduchon, and E. Mazet, Le Spectre D'une Variete Riemannienne, Lecture notes in Math 194, Springer, 1971.

[F] G. Folland, Spherical harmonic expansion of the Poisson-Szegö kernel for the ball, Proc. of the A. M. S., 47:2 (1975), pp. 401-408. 
[G] M. Gromov, Metric Structures for Riemannian and Non-Riemannian Spaces (with Appendices by M. Katz, P. Pansu, and S. Semmes), Birkhauser, 1999.

[GM] M. Gromov and V. D. Milman, A topological application of the isoperimetric inequality, Amer. J. Math., 105 (1983), pp. 843-854.

[H] S. Helgason, Groups and Geometric Analysis, Academic Press, 1984.

[K] T. KOORNWINDER, The addition formula for Jacobi polynomials and spherical harmonics, SIAM J. Appl. Math., 25:2 (1973), pp. 236-246.

[MS] V. D. Milman and G. Schechtmann, Asymptotic theory of finite dimensional normed spaces, Lecture notes in Math. 1200, Springer, 1985. 
